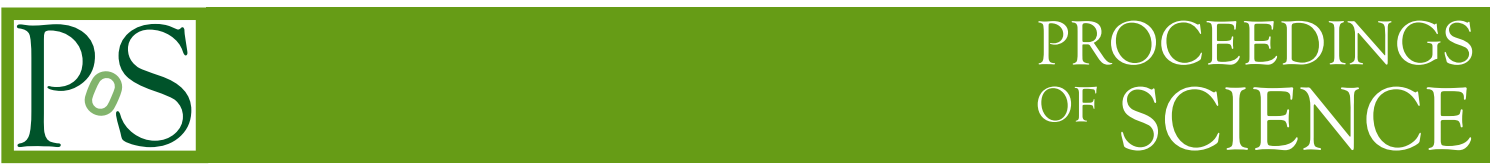

\title{
Results from Step I of MICE and the Physics Plan for Step IV
}

\author{
Yordan Karadzhov* on behalf of the MICE collaboration \\ University of Geneva \\ E-mail: yordan.karadzhovecern.ch
}

\begin{abstract}
The Muon Ionization Cooling Experiment (MICE) will demonstrate ionization cooling, an essential technology for a Neutrino Factory and/or Muon Collider, by measuring a 10-20\% reduction in the emittance of a muon beam. A realistic demonstration requires beams closely resembling those expected at the front-end of a Neutrino Factory, i.e. with large transverse emittance and momentum spreads. The MICE muon beam line at ISIS, RAL, was built to provide beams of different momenta and emittance so that the performance of the cooling channel can be fully explored. During the initial stage of MICE, a novel technique based on time-of-flight counters was used to establish that the beam emittances are in the range $0.7-2.8 \mathrm{~mm} . \mathrm{rad}$, with central momenta from $170-280 \mathrm{MeV} / c$, and momentum spreads of about $20 \mathrm{MeV} / c$. In the next stage of MICE the emittance of these beams will be increased by means of scattering from high- $\mathrm{Z}$ material and measured using magnetic spectrometers. Finally, low- $Z$ absorbers such as liquid hydrogen and $\mathrm{LiH}$ will be used to reduce the emittance of the beam and demonstrate the ionization cooling.
\end{abstract}

The European Physical Society Conference on High Energy Physics -EPS-HEP2013

18-24 July 2013

Stockholm, Sweden

\footnotetext{
* Speaker.
} 

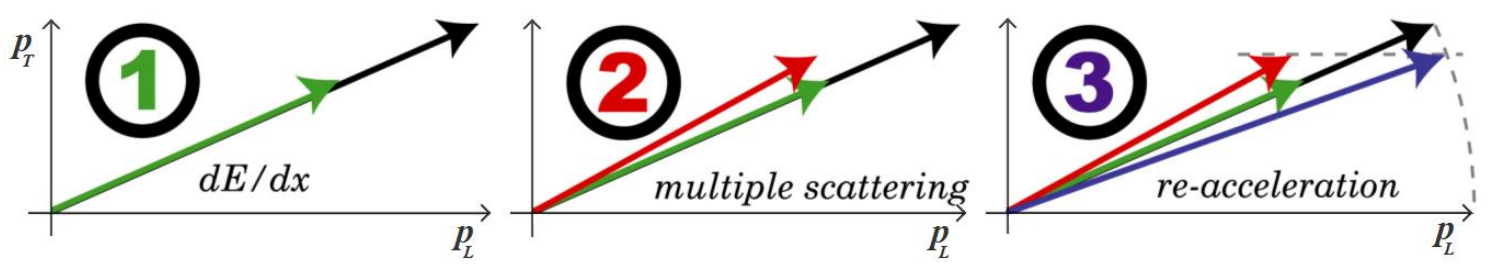

Figure 1: Ionization cooling principle: 1. Energy loss by ionization $(d E / d x) ; 2$. Heating from multiple scattering; 3. Longitudinal momentum restored by RF cavities.

\section{Ionization cooling}

Ionization cooling (Fig. 1) provides the only practical solution to prepare high brilliance beams necessary for a neutrino factory or muon colliders because it is fast enough to cool the beam within the muon lifetime.

To maximize the cooling effect we need material with low- $\mathrm{Z}$ and beam channel with low $\beta_{\perp}$.

\section{MICE}

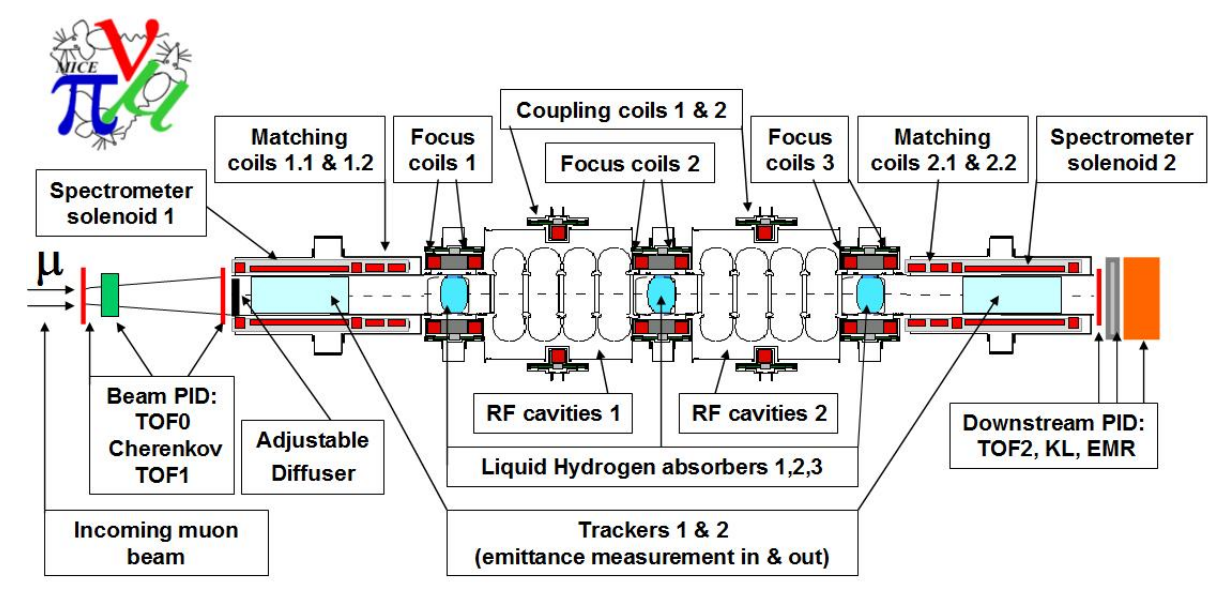

Figure 2: Layout of MICE.

The Muon Ionization Cooling Experiment (MICE) [1] (Fig. 2) is under development at the Rutherford Appleton Laboratory (UK). The goal of the experiment is to build a section of a cooling channel that can demonstrate the principle of ionization cooling and to verify its performance in a muon beam. The experiment will be assembled, tested and operated in steps (Fig. 3). Each step will validate different parts of the setup. Some steps have been removed from the original schedule. The final setup will be able to measure a $10 \%$ reduction in the emittance (transverse size) of the beam with a relative precision of $1 \%$.

MICE uses muon beams of limited intensity, where particles can be measured individually using scintillator-based detectors. The cooling cell in Step VI will include three Absorber-Focus Coils (AFC) and two RF Cavity-Coupling Coils (RFCC) modules. 

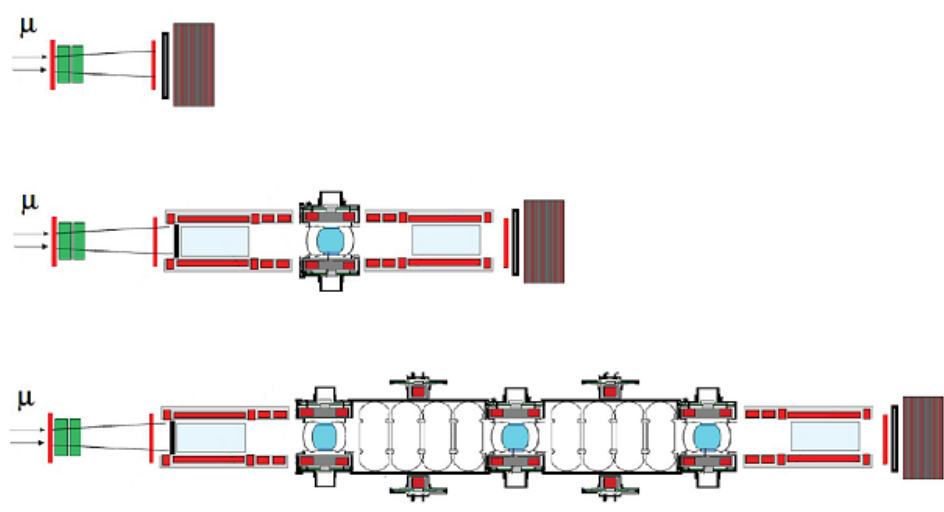

Figure 3: MICE schedule. Top: Step I; Middle: Stev IV; Bottom: Step VI.

In Step I (Fig. 3) of the experiment, the particle content of the muon beam has been measured by using time-of-flight detectors (TOF0, TOF1, TOF2), Cherenkov detectors and the KL (KLOE Light) calorimeter that provide precise muon, pion and electron identification. A time of flight detector timing resolution of $\approx 50$ ps was achieved [3].

In Step IV and VI (Fig. 3) two identical spectrometers and time measurements will be situated upstream and downstream of the cooling section and will measure the spatial coordinates and momentum $\left(x, y, p_{x}, p_{y}, p_{z}\right)$ of each muon.

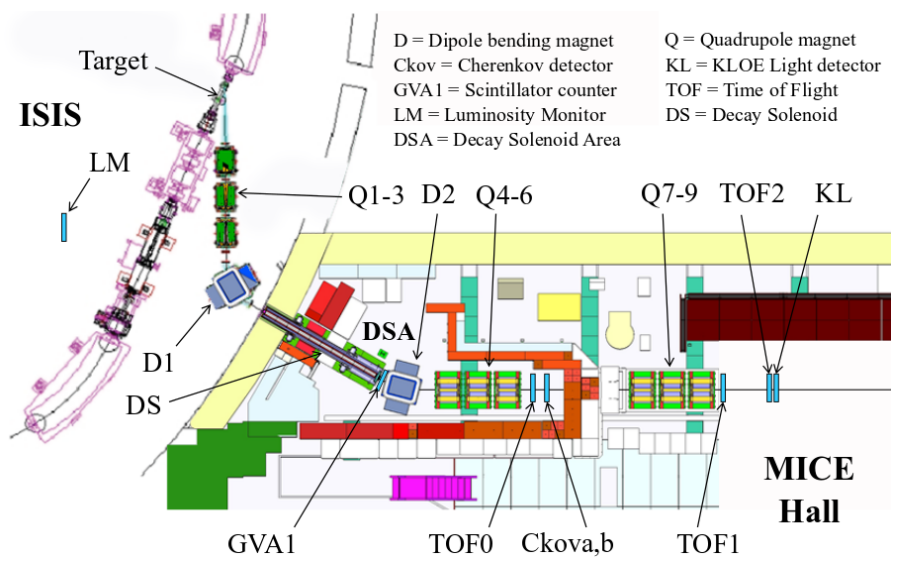

Figure 4: Top view of the MICE beam line with its instrumentation, as used in Step I.

\section{MICE beam line}

The MICE beam line (Fig.4) must generate several matched muon beams with different momenta and optical parameters at the entrance of the cooling channel. This is done using a titanium target which is dipped into the ISIS proton beam. The particles (mostly pions), resulting from interaction of the proton beam and the target, are captured and momentum-selected by the first 
dipole magnet (D1). After this a $5 \mathrm{~T}$ superconducting decay solenoid (DS) is used to contain and transport the pions and their decay muons. Another dipole (D2) then selects the final particles for propagation through the rest of the MICE beamline.

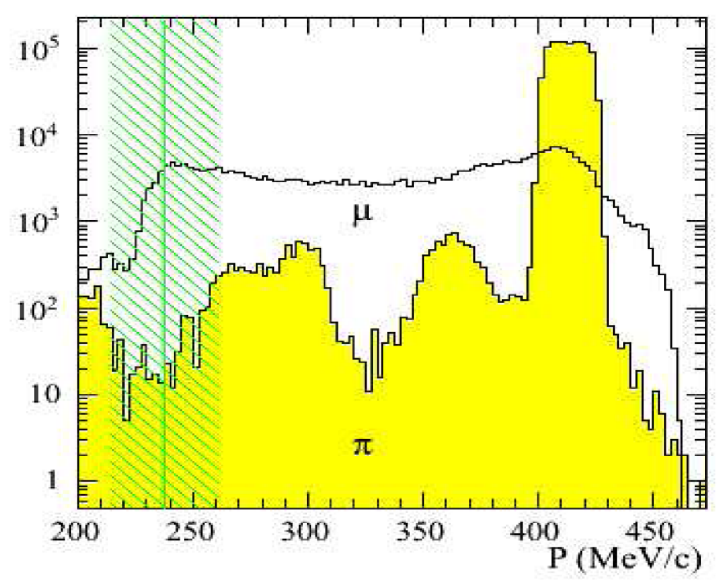

Figure 5: Simulation of the muon and pion spectra at the exit of the decay solenoid. Only high momentum pions survive. The green band shows the acceptance of $\mathrm{D} 2$, when tuned for backward-going muons.

The pion decays in flight provide a muon beam which has a large momentum spread at the exit of the decay solenoid. This is illustrated in Fig. 5. The maximum and minimum muon momenta are given by the decay muons flying forward and backward in the centre of mass system. Distinctly different distributions of particles can be produced by changing the nominal momentum, selected in the second dipole magnet.

The first possibility is to set the difference between the momenta selected in the first and the second dipole to be equal to the most probable energy losses of the particles in the materials between the dipoles. This type of beam is very useful for calibration of the detectors, as electrons, muons and pions fall into three well defined peaks in the time-of-flight spectrum with a narrow
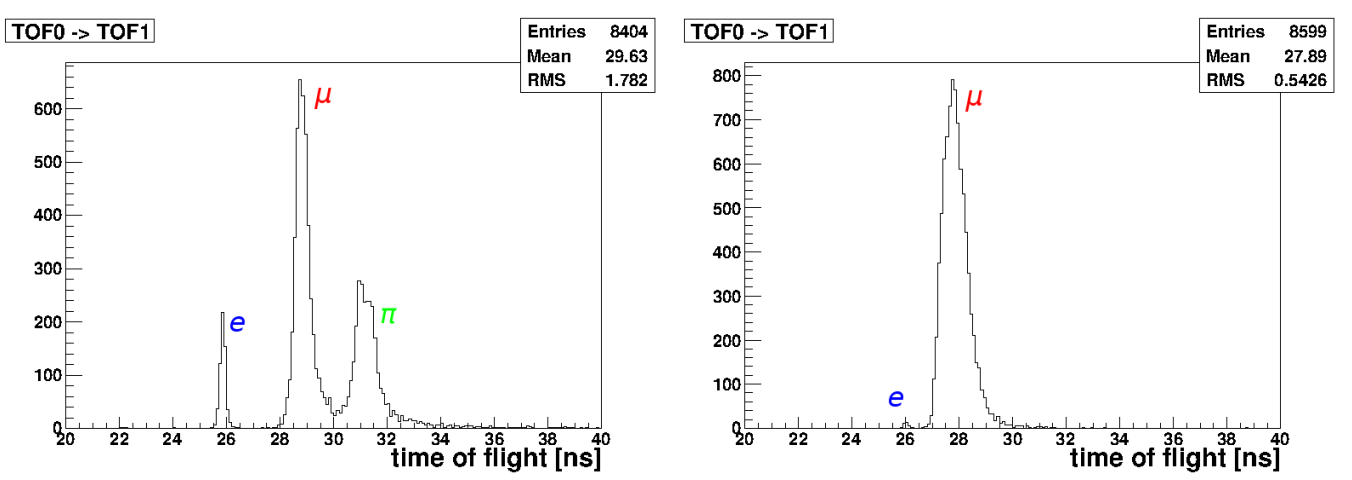

Figure 6: Time-of-flight distributions. Left: Low emittance beam for calibration of the detectors. Electrons, muons and pions fall into three well defined peaks. Right: High emittance muon beam with small contamination of electrons and pions will be used to demonstrate ionization cooling. 
(few \%) momentum spread (Fig. 6, right).

Another possibility is to select particles having momentum close to the minimum muon momentum. This will produce a muon beam with a small contamination from electrons and pions and a momentum spread of typically $25 \%$ (Fig. 6, left). Such a beamline configuration is usually called a muon beam and will be used to demonstrate ionization cooling.

\section{Step I results}

\subsection{Measurement of the muon rates}

Table 1: Muon rates ( $\mu / s$ per V.ms) for negative (top) and positive (bottom) polarities in the MICE beam line. Counts are normalized to the V.ms units used to characterize the induced ISIS beam loss. Errors are mainly due to the time-of-flight cuts used to define a muon.

\begin{tabular}{|l|c|c|c|}
\hline$\varepsilon_{N}$ & $\mu^{-}$rate $P_{Z}$ & $\mu^{-}$rate $P_{Z}$ & $\mu^{-}$rate $P_{Z}$ \\
$(\mathrm{~mm} \mathrm{rad})$ & $140 \mathrm{MeV} / \mathrm{c}$ & $200 \mathrm{MeV} / \mathrm{c}$ & $240 \mathrm{MeV} / \mathrm{c}$ \\
\hline 3 & $4.1 \pm 0.2$ & $6.3 \pm 0.2$ & $4.9 \pm 0.2$ \\
6 & $4.1 \pm 0.4$ & $4.8 \pm 0.2$ & $4.5 \pm 0.2$ \\
10 & $4.6 \pm 0.2$ & $5.4 \pm 0.2$ & $4.4 \pm 0.2$ \\
\hline \hline$\varepsilon_{N}$ & $\mu^{+}$rate $P_{Z}$ & $\mu^{+}$rate $P_{Z}$ & $\mu^{+}$rate $P_{Z}$ \\
$(\mathrm{~mm} \mathrm{rad})$ & $140 \mathrm{MeV} / \mathrm{c}$ & $200 \mathrm{MeV} / \mathrm{c}$ & $240 \mathrm{MeV} / \mathrm{c}$ \\
\hline 3 & $16.8 \pm 1.8$ & $33.1 \pm 3.2$ & $33.0 \pm 2.6$ \\
6 & $17.8 \pm 1.8$ & $31.0 \pm 2.0$ & $31.7 \pm 2.0$ \\
10 & $21.6 \pm 2.2$ & $34.0 \pm 2.5$ & $26.1 \pm 1.5$ \\
\hline
\end{tabular}

The number of muons per target dip (spill) in the MICE beam line as a function of ISIS beam loss has been studied. The observed particle rates in the TOF0 and TOF1 detectors were recorded and the measured time-of-flight was used to select good muon tracks [4]. The rates are found to be linear with the ISIS beam loss/target depth. The results are summarized in Table 1.

The number of muons per spill is presently limited by the tolerance of the irradiation caused in ISIS by protons and secondary particles produced in the MICE target. The rates obtained are sufficient to collect the $\sim 10^{5}$ muons necessary to perform a relative measurement of cooling with a precision of $1 \%$, in about one day.

\subsection{Emittance measurement}

In Step I of the experiment a novel single-particle method for emittance measurement has been developed [5]. The position and time measured by the two hodoscops TOF0 and TOF1 are used to reconstruct the beam emittance from a large sample of muons. The measured emittances and optical parameters of the beams are in good agreement with the simulations. In addition to this, the measured beams have been used as an input and propagated forward through the simulated cooling channel of Step VI. The observed reduction in emittance of the beam in this simulations was found to be bigger than $10 \%$. The results are summarized in Fig. 7, Fig. 8 and Fig. 9. 

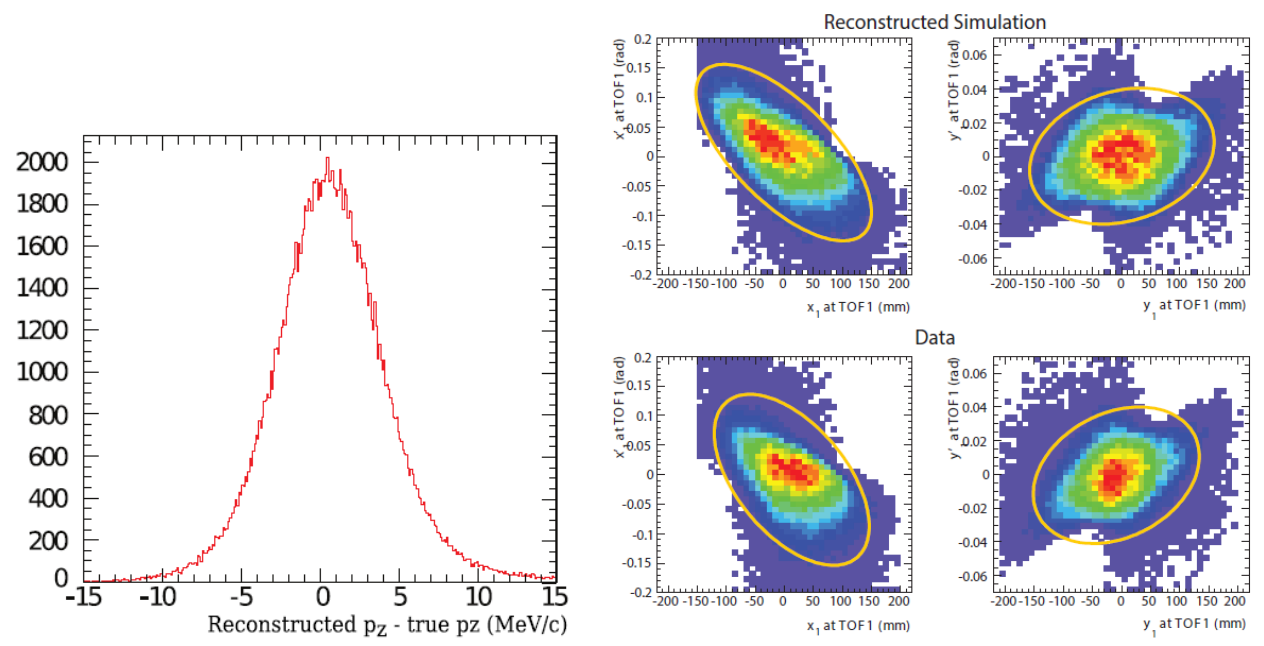

Figure 7: Left: The emittance reconstruction method achieves 3.7 MeV/c resolution for $240 \mathrm{MeV} / \mathrm{c}$ muons $(1.5 \%)$. Right: Horizontal $\left(x, x^{\prime}\right)$ and vertical $\left(y, y^{\prime}\right)$ trace space distributions at TOF1 for simulation (top) and data (bottom) for a nominal muon beam.
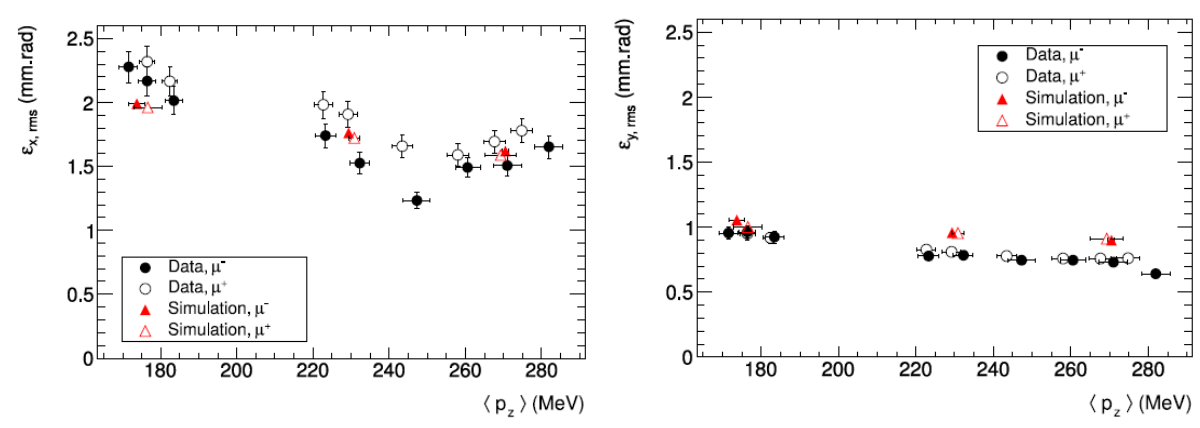

Figure 8: Beam emittance - MC vs. Data for various beam configurations. Left: Horizontal emittance. Right: Vertical emittance.

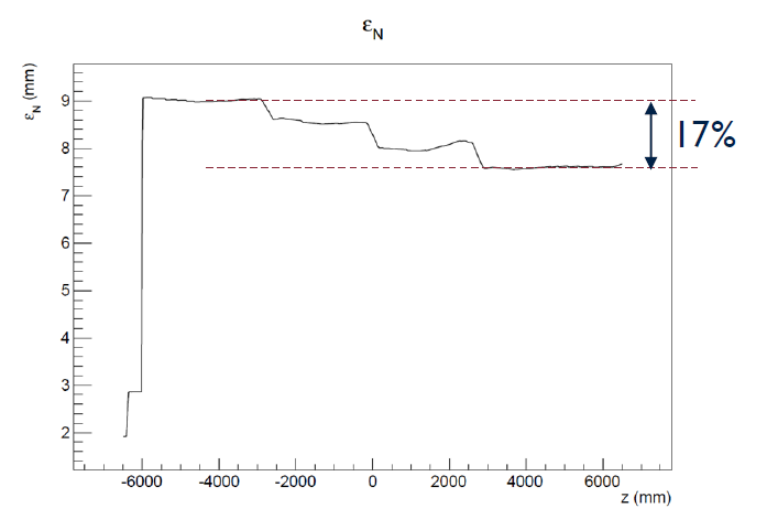

Figure 9: Expected Performance of the Cooling Channel. Measured beams are propagated forward through a full simulated cooling channel. 

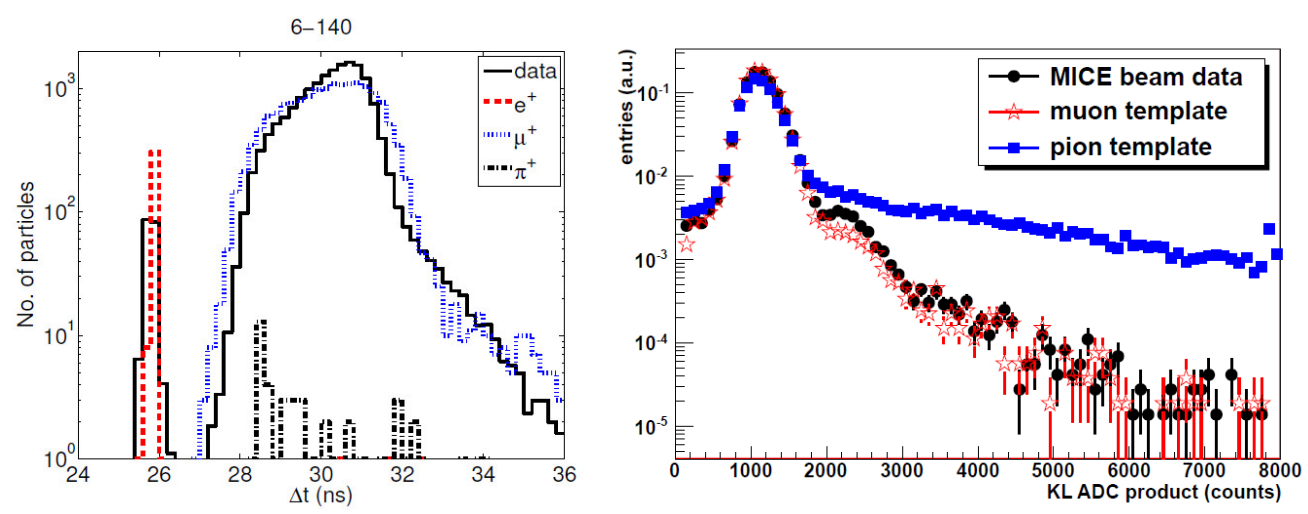

Figure 10: Left: Time-of-flight distributions between TOF0 and TOF1 for data and Monte Carlo simulation. $6 \pi \mathrm{mm} \times \mathrm{rad}$ positive muon beams with nominal beam momentum $p_{\mu}=140 \mathrm{MeV} / \mathrm{c}$. Right: Muon and pion templates from calibration runs, compared to MICE muon beam data.

\subsection{Study of the pion contamination in the muon beam}

A low pion contamination in the muon beam is an essential requirement for a precise measurement of ionisation cooling. The pion contamination at the entrance of the cooling channel has been measured statistically using the distribution of pulse heights in KL (Fig. 10, right). It is found to be $1 \%$ or below [6], as expected from Monte Carlo simulations (Fig. 10, left).

\section{Physics plan for Step IV}

The physics plan for Step IV includes measurements of the equilibrium emittance of a given absorber and for a given beta function and measurement of 6D emittance change, as well as precision measurements of multiple scattering. Step IV is expected to be ready to take data in 2015 [7].

\section{References}

[1] MICE web site http://mice.iit.edu contains detailed information about the experiment.

[2] MICE proposal, MICE note 21, (2003)

[3] MICE collab. , The MICE Muon Beam on ISIS and the beam-line instrumentation of the Muon Ionization Cooling Experiment, JINST 7 (2012) P05009, arXiv:1203.4089v2

[4] A. Dobbs, K. Long, and A. Alekou,'The MICE muon beamline and induced host accelerator beam loss", Proceedings 2011 Particle Accelerator Conference (PAC'11), 28 March-1 April 2011, New York, 2011, MOP021.

[5] MICE collab. Characterisation of the muon beams for the Muon Ionisation Cooling Experiment, Eur. Phys. J. C (2013) 73: 2582, arXiv:1306.1509

[6] Measurement of the pion contamination in the MICE beam , MICE note 416, (2013)

[7] Progress towards completion of the MICE demonstration of ionisation cooling of muons, these proceedings. 